\title{
Light Scatter From the Central Human Cornea
}

\author{
GEORGE T. H. SMITH, NICHOLAS A. P. BROWN, G. ADRIEN SHUN-SHIN \\ Oxford
}

\begin{abstract}
Summary
Light scatter from the central human cornea was measured in 60 eyes of 60 patients using computerised image analysis of Scheimpflug slit image photographs. There was no correlation between the amount of scatter and the age of the patients (correlation $0.035, p=0.79$ ). In addition it was found that light was scattered more at the anterior and at the posterior surfaces of the cornea than from the stroma.
\end{abstract}

Scheimpflug photography is a well recognised technique for examining slit images of the anterior segment of the eye. ${ }^{1,2}$ A sagittal section of the anterior segment is obtained, making it possible to examine the light scattering properties of the lens and cornea using computerised linear scanning densitometry. Linear scanning densitometry has been used extensively to study light scatter in the lens $s^{2,6,7,8}$ and would seem well suited to study the light scattered by the cornea.

Previous authors have used other techniques to examine the amount of light either transmitted or scattered by the human cornea. Olsen ${ }^{3}$ found that corneal light scatter increased with age, in vivo, and Lerman ${ }^{4}$ in an in vitro study stated that there was a reduction in transmission with age, possibly as a result of the accumulation of fluorescent chromophores with advancing years. Van Best, ${ }^{5}$ however, found no difference in the amount of light transmitted at different ages using cadaver eyes.

The aim of the present study was to examine the light scattering properties of the human cornea, in vivo, using computerised linear scanning densitometry of Scheimpflug photographs.

\section{Materials and methods}

In a retrospective study, 60 Scheimpflug photographs from the eyes of 60 subjects were analysed using computerised linear scanning densitometry. The right eye was chosen for analysis in all except 14 cases where only the photogaphs from the left eye were available. The ages of the subjects ranged from seven to 88 years (mean 44.52 ) of which there were 33 males and 27 females. The photographs were taken from our library of negatives and were stored alongside sheets containing clinical information about the subjects. These negatives had been made on Ilford XP1 400 film using the Brown slit image camera, ${ }^{1}$ which has an unfiltered xenon flash light source. The photographs had been taken to study lens changes in the subjects who had various types of cataract. Photographs of patients who had any clinical corneal abnormality were excluded as were the photographs of diabetics.

The photographic negatives were optically focused onto a CCD (charge couple device) using a modified Illumitran (slide copier), and the images were then read into an Epson PC AX (10 MHZ) desktop microcomputer by means of a frame store.

The analysis of the photographs was performed using the technique as described by Sparrow $^{6}$ for examining the light scattering properties of the human lens. The technique

From: Clinical Cataract Research Unit, Nuffield Laboratory of Ophthalmology, Oxford.

Correspondence to: George T. H. Smith FRCS, Clinical Cataract Research Unit, Nuffield Laboratory of Ophthalmology, Walton Street, Oxford OX2 6AW. 


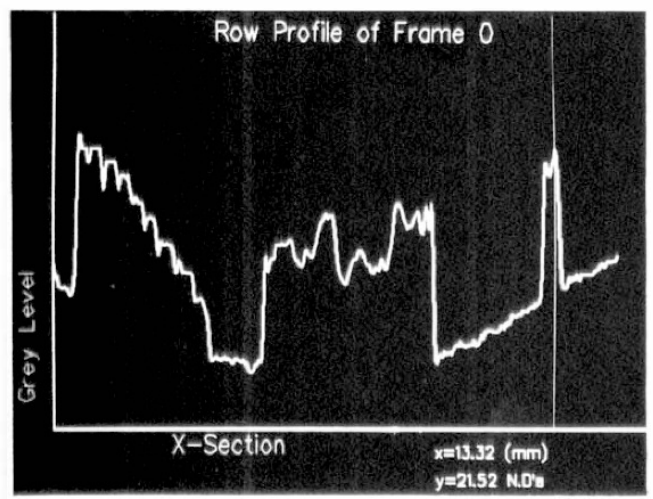

Fig. 1. The area of interest could be identified on the densitogram by means of a cursor (seen here positioned on the peak of the corneal spike).

measures the density, relative to a neutral density stairase, of an axial strip of photograph measuring five pixels on either side of a central line. This is equivalent to a central strip of cornea measuring $0.26 \mathrm{~mm}$. Both the maximum peak and the total area under the densitogram of the corneal spike (area planimetry) were taken as parameters for assessing the amount of scatter.

Dimensions were measured using the axial linear density trace of the Scheimpflug images. Points for measurement were identified from the trace, the cursor being simultaneously displayed on both the computer screen, which displayed the Scheimpflug photograph and the black and white video screen, which displayed the axial densitogram, allowing judgements to be confirmed on the image itself (see Figs. 1 and 2). The corneal thickness was also measured. The posterior peak of increased scatter was not constant and tended to be less than the anterior peak which was always present. We therefore chose not to measure the posterior peak.

In order to minimise observer bias the photographs were analysed before the clinical information was recorded so that the examiner did not know the patient details while carrying out the measurements.

\section{Results}

There was no correlation between the age of the subjects and the amount of corneal scatter (correlation $0.03, \mathrm{p}=0.79$ for maximum peak and $0.030, \mathrm{p}=0.820$ for area under corneal peak) (see Figs. 3 and 4). In addition the amount of scatter was not uniform throughout the thickness of the cornea and the intensity of light scattered was greater at the anterior and posterior surfaces than at its centre (see Fig. 5). No relationship was demonstrated between the age of the subjects and the corneal thickness.

\section{Discussion}

Linear scanning densitometry of Scheimpflug photographs is a well recognised technique for examining the light scattering properties of the human lens, in vivo. ${ }^{2,6,7,8}$

In this study we applied the same technique directly to measure light scattered by the cornea.

It might have besen expected that the light scattering properties of the human cornea would increase with age as clinically, old corneas tend to appear less transparent than young ones. It should be remembered however that the changes that occur with age are more apparent in the corneal periphery than at its centre. ${ }^{9}$ In this study it was only the centre of the cornea that was measured.

Olsen, ${ }^{3}$ using a technique which employed a fibre optic probe and a photomultiplier, compared the amount of angular scatter from the central cornea to a standard sodium fluorescein solution. He found that there was a significant increase in the amount of light scatter with age, in vivo. This was independent of the corneal thickness which, as in our study, did not alter with age and he postulated that it was as a result of the spacing of the collagen fibres becoming less regular with advancing years. His technique, however, measured scatter from the full corneal thickness and was not able to differentiate scatter from the separate corneal layers. In his technique the source light was blue in the range of 400 to $500 \mathrm{~nm}$, whereas we used a xenon flash tube which had a much wider spectrum.

Lerman $^{4}$ also discovered that there was a reduction in transmissivity with advancing years, in vitro, and he attributed this to an accumulation of a small amount of fluorescent chromophores although he found this to be much less important than in the lens. It may be relevant to the study by Olsen ${ }^{3}$ in that it is 


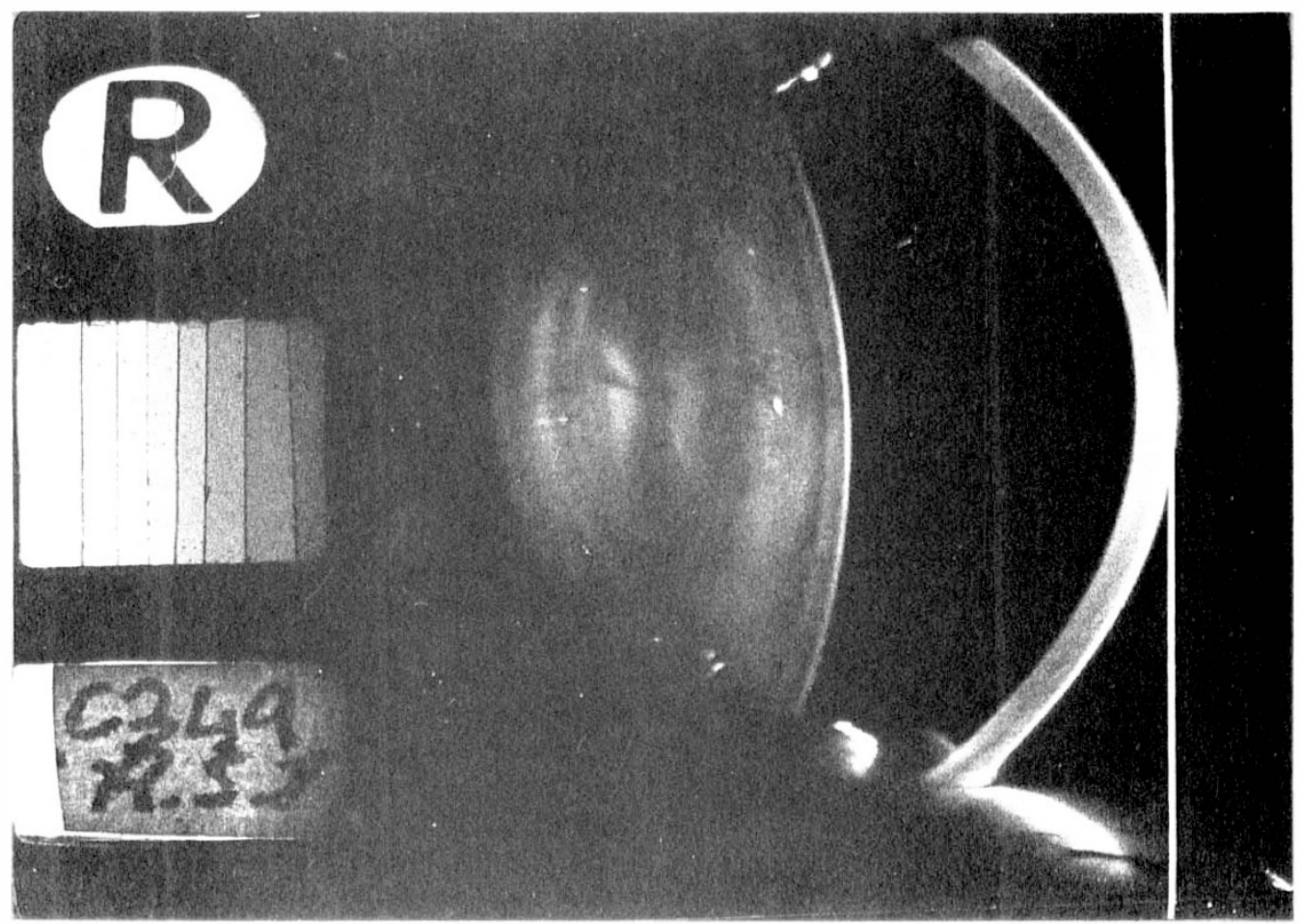

Fig. 2. The cursor was simultaneously displayed on the video screen showing the Scheimpflug image enabling a more precise comparison between the densitogram and the area on the image that it represented.

the blue end of the spectrum which induces fluorescence and this could account for his finding increasing values with age.

In the study by Van Best, ${ }^{5}$ using cadaver eyes, it was shown that corneal transmissivity did not decrease with age and this would tend to concur with our results.

Light scattering from the corneal stroma is thought to increase when the orientation of

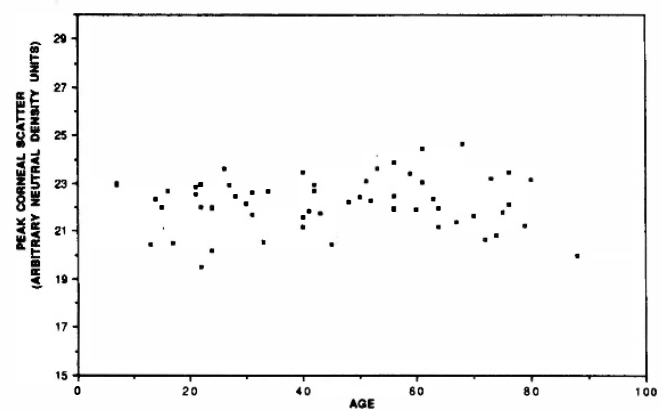

Fig. 3. No correlation could be found between the age of the subjects and the maximum peak of the corneal spike on the densitogram. the collagen fibrils becomes altered. ${ }^{3}$ Goldmann ${ }^{10}$ believes that light scattering in the oedematous cornea occurs when there are fluctuations in its refractive index which are distributed over distances which are larger than one half of the wavelength of light. The critical value for this distance is around $2000 \mathrm{~A}$. It is known that the pump activity of the human endothelium diminishes with age

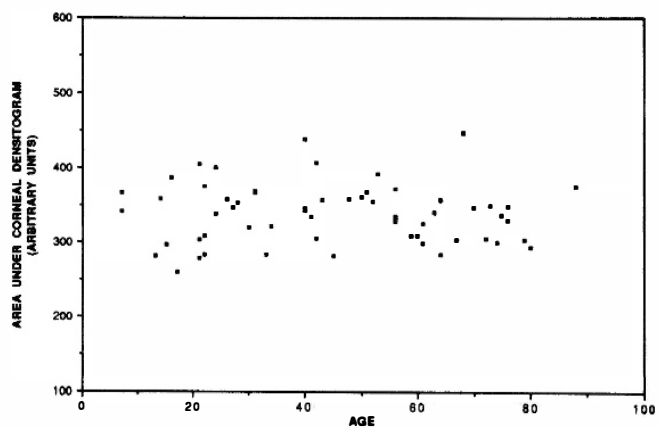

Fig. 4. No correlation could be found between the age of the subjects and the area under the corneal spike of the densitogram. 


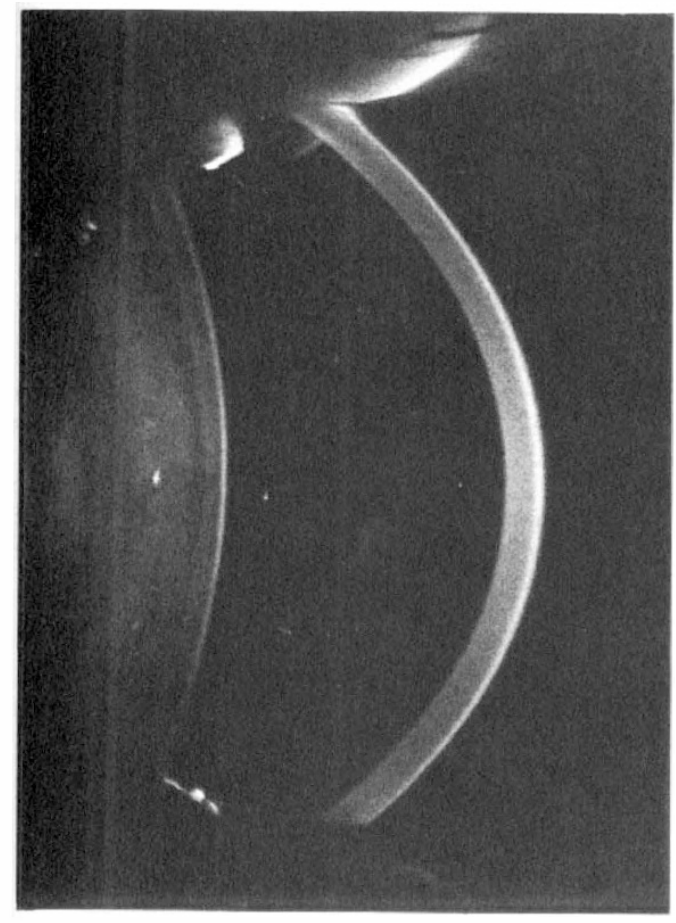

Fig. 5. Scheimpflug photograph of cornea showing that more light is scattered from the anterior and posterior corneal surfaces than from the stroma.

and it might be expected that this would interfere with the transparency of the cornea. It has also been shown, however, that this is at least partly compensated for by the fact that the barrier function of the endothelium and its ionic permeability are increased with age. ${ }^{11}$

It might be criticised that the sample chosen for this study was poorly representative of the population as a whole as all of the subjects had cataracts. This would have to be conceded, however none of the subjects had any clinically detectable corneal pathology and it was thought unlikely that the cataracts would bear any relationship to the amount of corneal light scatter. Olsen ${ }^{3}$ in his study also included patients with cataracts.

It was interesting to note that the amount of scatter was not uniformly distributed throughout the cornea and that more scatter occurred at its anterior and posterior surfaces than at its centre. Presumably most scatter was occurring at interfaces where there is a large change in refractive index, i.e. the epithelial and endothelial surfaces and also at Bowman's membrane which would contribute to the anterior peak. This has been observed by previous workers. ${ }^{12,13,14}$ Lindstrom $^{14}$ stated that the main contribution to the integrated scattered intensity from the rabbit cornea came from the regions close to the epithelium and endothelium. He also observed that more scatter occurred at the anterior than the posterior side of the cornea and attributed this to the collagen fibres being more irregularly orientated in the anterior than the posterior stroma. Previous measurements of this, however, have only been made on in vitro preparations in rabbits ${ }^{13,14}$ and although it has been noticed clinically using the slit lamp, ${ }^{12}$ to our knowledge, it has never been quantified in vivo.

In conclusion, we feel that computerised linear scanning densitometry of Scheimpflug photographs is a useful technique for examining the light scattering properties of the cornea as well as the lens and that it therefore has the potential to be used in the evaluation of corneal scarring and to monitor the progress of corneal oedema.

Recently, a technique has been developed at Oxford which enables photographs to be taken and fed directly into a computer by means of a CCD camera and therefore dispense with film altogether. This will greatly facilitate the follow up of patients with corneal pathology since a result would be available immediately without having to wait for film to be developed thus allowing treatment to be monitored. Another useful application of this technique may be in the assessment of iritis since it was shown by Anjou and Kra$\mathrm{kou}^{15}$ that anterior chamber light scattering increased in patients with this condition. Our technique offers the facility to measure this objectively in a matter of minutes and could prove a valuable method of assessing antiinflammatory treatment.

\section{References}

${ }^{1}$ Brown NAP: Slit image photography. Trans Ophthalmol Soc UK 1969, 89: 387-408.

${ }^{2}$ Brown NAP: Quantitative slit image photography of the lens. Trans Ophthalmol Soc UK 1972, 92: 303-17.

${ }^{3}$ Olsen T: Light scattering from the human cornea. Invest Ophthalmol Vis Sci 1982, 23: 81-6. 
${ }^{4}$ Lerman S: Biophysical aspects of corneal and lenticular opacity. Curr Eye Res 1984, 3: 5-14.

${ }^{5}$ Van Best JA: Corneal transmission in whole human eyes. Exp Eye Res 1988, 46: 765-8.

${ }^{6}$ Sparrow J: The lens in diabetes. D.Phil. Thesis, Oxford, 1989: 97-100.

${ }^{7}$ Hockwin $\mathrm{O}$ and Laser $\mathrm{H}$ : Image analysis of Scheimpflug negatives: comparative quantitative assessment of the film blackening by area planimetry and height measurements of linear densitograms. Ophthalmic Res 1988, 20: 99-105.

${ }^{8}$ Hockwin O, Dragomirescu V, Laser H: Measurement of lens transparity and its disturbance by densitometic image analysis of Scheimpflug photographs. Graefes Arch Clin Exp Ophthalmol 1982, 219: 255-62.

${ }^{9}$ Ansons AM and Atkinson PL: Corneal Mosaic Patterns-Morphology and Epidemiology. Eye 1989, 3: 811-15.
${ }^{10}$ Goldman JN, Benedek GB, Dohlman CH, Kravitt B: Structural alterations affecting transparency in swollen human corneas. Invest Ophthalmol 1968, 7: 501.

${ }^{11}$ Wigham CG and Hodson SA Physiological changes in the cornea of the ageing eye. Eye 1987, 1: $190-6$.

${ }^{12}$ Duke-Elder S and Wybar KC: System of Ophthalmology, CV Mosby Co, St Louis. 1961: Vol 2: 100.

${ }^{13}$ McCally RL and Farrell RA: The depth dependance of light scattering from the normal rabbit cornea. Exp Eye Res 1976, 23: 69

${ }^{14}$ Lindstrom JL, Feuk T, Tengroth B: The distribution of light scattered from the rabbit cornea. Acta Ophthalmol 1973, 51: 656-69.

${ }^{15}$ Anjou CN and Krakau CET: A photographic method for measuring the aqueous flare of the eye in normal and pathological conditions. Acta Ophthalmol 1960, 38: 178-224. 